\title{
Infodemic in Pandemic of COVID-19
}

\author{
Mohammadhadi Abbasi ${ }^{1}$, Seyed Mohammad Hashemi-Shahri ${ }^{1}$, Alireza Ansari-Moghaddam ${ }^{1}$, \\ Mohammad Khammarnia (Did ${ }^{1,}{ }^{*}$, Farid Najafi ${ }^{2}$ and Fatemeh Setoodehzadeh (D) ${ }^{1}$ \\ ${ }^{1}$ Health Promotion Research Center, Zahedan University of Medical Sciences, Zahedan, Iran \\ ${ }^{2}$ Research Center for Environmental Determinants of Health (RCEDH), Health Institute, School of Health, Kermanshah University of Medical Sciences, Kermanshah, Iran \\ Corresponding author: r, Health Promotion Research Center, Zahedan University of Medical Sciences, Zahedan, Iran. Email: m_khammar1985@yahoo.com
}

Received 2020 May 27; Revised 2020 May30; Accepted 2020 May 31.

Keywords: Iran, Infodemic, COVID-19

\section{Dear Editor,}

Viruses are microscopic parasites and generally much smaller than bacteria. They lack the capacity to thrive and reproduce outside of a host. Predominantly, viruses have a reputation for being the cause of infection (1). However, only a small number of microbes, parasites, and other pathogens in such a way need living organisms as their hosts to survive. In this regard, human communication and more importantly, the lifestyle of individuals are very important elements in the spread of the virus and completion or interruption of the transmission cycle. The same is true and can be applied in the case of the COVID-19 virus.

In the first several days of the COVID-19 outbreak in China in 2020, educational activities were started exclusively by China's health care systems (2). However, unlike previous epidemics, a combination of biomedical, social, media, communication, economic, and even political factors caused the journalistic, media, and security dimensions of this epidemic to even overcome its biomedical aspects (3). Accordingly, the unrecognizability of the new virus to the medical community, the rapid rate of its transmission, and the unknown behavior of the new virus have resulted in a wide wave of media activities around this pandemic (4). In addition, the outbreak of the virus in Wuhan and China, which is located in the opposite pole of the United States, has also strengthened the media and security aspects of the epidemic.

The coronavirus pandemic continues to reveal the economic dimension of the epidemic, and the extent of the virus's impact on the global, regional, national, and local economies is much broader and deeper than initial predictions (5). Consequently, the combination of these factors and many other psycho-social and even ideological variables and the widespread access of the world commu- nity to the media, especially its electronic and digital types, has produced a huge amount of data and information including those correctly and incorrectly published and republished (6). Accordingly, more than millions of messages, clips, and educational content related to COVID-19 are published on communication networks every day (7). This huge amount of information has become a public concern worldwide. In the meantime, the published information includes a wide range, which can be prominently characterized in the following four categories:

1) Accurate and helpful information

2) Correct but ineffective information

3.) Incorrect and problematic information

4) Incorrect but safe information

People could clearly sort the received information into one of the four aforementioned classes, and in order to do so, they need to have a set of skills. Principally, health literacy, and media literacy are the most important skills in this regard. To achieve media literacy, it is suggested that people have to identify the source of data as soon as they receive any information, and thus increase the probability of success in analyzing and classifying the evidence based on the goals and motivation of the source (8).

The dissemination of incorrect information related to COVID-19 is generally done in two organized and unorganized dimensions.

1) Non-organized dissemination of incorrect information:

A) Irresponsible and uninformed people about COVID19 who feel that they have to share their information with others simply because of human issues.

B) People who seek to be considered, due to psychological reasons, try to republish or even produce educational content, and this information is generally incorrect.

2) Organized dissemination of incorrect information: 
A) Distribution of incorrect information for economic purposes; for example, selling industrial alcohol instead of ethanol in Iran to prevent disease resulted in 796 deaths from February 20 to April 25, 2020. Another example of this is the promotion of a special type of food supplement by celebrities in cyberspace.

B) Broadcasting of incorrect information for political purposes. Usually, countries, organizations, and political factions use every opportunity to knock out each other, and unfortunately, some of them use the COVID-19 epidemic to accelerate and facilitate the achievement of their goals.

C) Spreading of symbolic information for ideological purposes.

Nowadays, in the global village in which all countries have many connections and interactions with each other, the outbreak of the disease in one part of the world may possibly affect other parts of the globe. On the other hand, the infodemic at the international level could prolong the passage of the corona pandemic, and it could have a major impact on the global economy and people's quality of life. Therefore, global management for overcoming a pandemic especially the dissemination of infodemic resources and rumors, is necessary. In this way, regardless of any political and biased views, the world community could control the disease and reduce pressure on health systems, and health care staff. In this regard, the WHO launched a TikTok account as part of its efforts to reduce or cut coronavirus misinformation and to get and prepare accurate information concerning the novel coronavirus to people online. According to the MIT Technology Review, WHO and the Centers for Disease Control and Prevention have spent the last couple of weeks of the outbreak fighting misinformation regarding the virus on platforms like Facebook, Twitter, Tencent, and TikTok (9). In order to prevent the spread of misinformation at the international and regional levels, it is suggested that the provision of information should be taken seriously in order to promote preventive behaviors and avoid misbehavior, which contributes to the spread of the virus in society.

\section{Footnotes}

Authors' Contribution: All authors have contributed to all sections of the manuscript preparation.

Conflict of Interests: The authors have no conflict of interest.

Funding/Support: This study was conducted without any funding.

\section{References}

1. Wolf K. Fish viruses and fish viral diseases. Cornell University Press; 2019.

2. Wang C, Cheng Z, Yue X, McAleer M. Risk management ofCOVID-19 by universities in China. Multidisciplinary Digital Publishing Institute; 2020.

3. McCloskey B, Zumla A, Ippolito G, Blumberg L, Arbon P, Cicero $A$, et al. Mass gathering events and reducing further global spread of COVID-19: a political and public health dilemma. Lancet. 2020;395(10230):1096-9.

4. Sohrabi C, Alsafi Z, O'Neill N, Khan M, Kerwan A, Al-Jabir A, et al. World Health Organization declares global emergency: A review of the 2019 novel coronavirus (COVID-19). Int J Surgery. 2020.

5. Fernandes N. Economic effects of coronavirus outbreak (COVID-19) on the world economy. SSRN. 2020.

6. Zheng Y, Goh E, Wen J. The effects of misleading media reports about COVID-19 on Chinese tourists' mental health: a perspective article. Anatolia. 2020:1-4.

7. Rajput NK, Grover BA, Rathi VK. Word frequency and sentiment analysis of twitter messages during Coronavirus pandemic. Preprint. 2020

8. Cinelli M, Quattrociocchi W, Galeazzi A, Valensise CM, Brugnoli E, Schmidt AL, et al. The covid-19 social media infodemic. Preprint. 2020

9. Oxford Analytica. Misinformation will undermine coronavirus responses. Emerald Expert Briefings. 2020;(oxan-db). 